\title{
La territorialización de las políticas públicas en Argentina. Un estudio acerca del CPRES en el área metropolitana
}

\author{
Giselle González
}

\begin{abstract}
Resumen
El artículo se propone describir la función normativa y el rol efectivo del Consejo de Planificación Regional de la Educación Superior (CPRES), desde la sanción de la Ley 24.521/95 hasta 2010. En particular, se describe cómo se piensa al CPRES en el marco de la implementación de la Ley, qué modelo de gestión se esperaba instalar con su incorporación y cuál se puso en funcionamiento finalmente. Se examinó el área metropolitana porque reúne a la mayoría de los representantes de las universidades nacionales con mayor tamaño y tradición, las universidades privadas y los institutos de educación superior no universitaria (IESNU). Se analizaron fuentes documentales y entrevistas en profundidad a informantes clave del ámbito académico, técnicos y políticos que participaron en el Programa de Reforma de la Educación Superior, iniciado en 1995. Con base en este análisis se evidenció que el CPRES se constituye como una estructura de autoridad de nivel intermedio con capacidad jurídico-institucional para planificar, coordinar y regular los procesos de implementación de las políticas en el marco de un contexto sistémico complejo y de alta diferenciación institucional. Sin embargo, aún no logran constituirse como efectivas autoridades de implementación.
\end{abstract}

Palabras clave: gobierno, gestión, poder, implementación, educación superior.

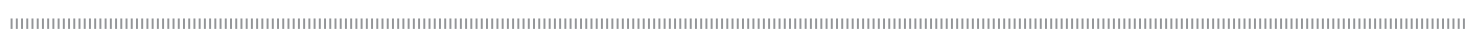

Giselle González

ggonzalez@udesa.edu.ar

Licenciada en Ciencia Política por la Facultad de Ciencias Sociales de la Universidad de Buenos Aires. Magister en Educación con orientación en Gestión Educativa y Candidata a Doctora en Educación con Beca de Iniciación de CONICET en la Universidad de San Andrés. Docente asistente en el seminario "Introducción a la Gestión Educativa" del Programa de Maestría en Educación dictado en la Universidad de San Andrés. 


\title{
A territorialização das políticas públicas na Argentina. Um estudo sobre o CPRES na área metropolitana
}

\section{Resumo}

O artigo se propõe descrever a função normativa e o papel efetivo do Conselho de Planejamento Regional de Educação Superior (CPRES), desde a promulgação da Lei 24.521/95 até 2010. Particularmente, descreve como é pensado o CPRES no marco da implementação da Lei, que modelo de gestão se esperava instalar com a sua incorporação, e finalmente qual foi o que ficou em funcionamento. Examinou-se a área metropolitana porque reúne à maioria dos representantes das universidades nacionais de maior porte e tradição, as universidades privadas e os institutos de ensino superior não universitário (IESNU). Analisaram-se fontes documentais e entrevistas em profundidade a informantes chave do âmbito acadêmico, técnicos e políticos que participaram do Programa de Reforma da Educação Superior, iniciado em 1995. Com base nesta análise se evidenciou que o CPRES constitui-se como uma estrutura de autoridade de nível médio com capacidade jurídico-institucional para planejar, coordenar e regular os processos de implementação das políticas no marco de um contexto sistêmico complexo e de alta diferenciação institucional. Entretanto, ainda no conseguem se constituir como efetivas autoridades de implementação.

Palavras chave: governo, gestão, poder, implementação, educação superior.

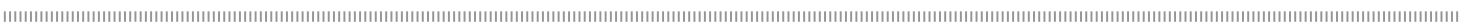

The territorialization of public policies in Argentina. A study on CPRES in the metropolitan area

\begin{abstract}
The article is aimed at describing the regulatory function and effective role of the Regional Planning Council for Higher Education (CPRES for its acronym in Spanish), since the passing of Law 24.521/95 until 2010. In particular, it describes thoughts about CPRES in the framework of the enforcement of said Law, expectations related to the administrative model arising from its implementation and the real model that was finally put into practice. The metropolitan area was chosen because it gathers most of the representatives of the largest and most traditional national and private universities, and the non-university higher education institutions (IESNU for its acronym in Spanish). Documentary sources and in-depth interviews with key informants of the academic, technical and political spheres involved in the Higher Education Reform Program initiated in 1995 were analysed. The analysis evidenced that CPRES is set up as an authority structure of intermediate level with legal institutional capacity to plan, coordinate and regulate the processes for the implementation of the policies within the framework of a complex systemic context and highly differentiated institutions. However, they have not managed to set themselves up as effective authorities of implementation.
\end{abstract}

Key words: government, administrative, power, implementation, higher education.

Recepción: 30/07/10. Aprobación: 01/09/10. 


\section{Introducción}

Durante las últimas décadas, en la mayoría de los países latinoamericanos, se han generado importantes transformaciones que han modificado la organización y la forma de gestionar los sistemas educativos. Dichos cambios significaron una fuerte redefinición de los roles que hasta ese momento habían desarrollado cada uno de los actores (individuales o colectivos, estatales o sociales) involucrados en la gestión, provisión y regulación de los servicios educativos. Uno de los elementos más visibles de esta redefinición de roles puede notarse claramente cuando se analiza y compara el rol que hasta hace relativamente poco tiempo desempeñaron los Estados nacionales y las nuevas responsabilidades que a partir de las reformas debieron asumir las instancias subnacionales de gobierno (regionales o locales) en materia educativa (Gvirtz y Dufour, 2008; Bobbio, 1991; Cabrero, 2006; Acuña y Repetto, 2006; Aguilar, 2007; Repetto, 2004). En cuanto a la regulación, los sistemas educativos atravesaron, en este periodo, por un doble proceso: por un lado, hubo una disminución de la cantidad de campos regulados por los Estados nacionales y, por otro, se dio un cambio en la naturaleza de las regulaciones, pasando éstas de regular procesos a regular también resultados. Finalmente, las variaciones en el campo de la provisión, tanto en la regulación como en el financiamiento, crearon las condiciones de existencia y posibilidad para que nuevos actores estatales y no estatales intervengan en la provisión de servicios educativos (Gvirtz y Dufour, 2008).

Argentina no ha sido excepción en referencia a los procesos reseñados. La Ley 24.521/95 de Educación Superior introduce un nuevo actor en el gobierno del sistema educativo. El Consejo de Planificación Nacional de la Educación Superior (CPRES) se piensa como un actor de nivel intermedio con capacidad para la elaboración e implementación de políticas educativas. Se crea para planificar, coordinar y regular el quehacer integral del sistema de educación superior. Sin embargo, en la actualidad parece cumplir otras funciones diferentes de las definidas en la norma que le da origen.

\section{EI CPRES como objeto de estudio}

El estudio propuesto se inscribe en el marco de los estudios sobre el surgimiento de nuevos actores e instituciones en el sistema de educación superior. Se abordó desde dos líneas analíticas. Desde la perspectiva neoinstitucional (North, 2001; Powell y DiMaggio, 1991; March y Olsen, 1997), se sustentan los conceptos e ideas que se incorporan para comprender el rol que desempeñan los actores de nivel intermedio en la implementación de políticas públicas en el campo de la educación superior.

Se plantea el problema del gobierno del sistema de educación superior, en general, y de los cambios que introduce la territorialización de las políticas públicas en dicho campo, y la organización del gobierno como una estructura multinivel, en particular. Esto supone la consideración de estructuras de autoridad y no sólo actores tomados individualmente. El término estructuras de autoridad refiere al "conjunto de los roles o modelos regularizados de comportamiento y de expectativas acerca del modo en que habrán de comportarse los que ocupan posiciones especiales en la sociedad y el modo en que los demás deben obrar frente a ellos" (Morlino, 1985: 39).

estructuras de toma de decisiones o de aplicación de estas últimas. En ambos casos, son las normas formales (Constitución, leyes, decretos, etcétera) las que determinan cuáles son esas estructuras de autoridad y las que regulan su ámbito competencial, quienes son las personas autorizadas para ocuparlas y bajo qué procedimientos, y cuáles son las funciones correspondientes a cada una de ellas (Morlino, 1985: 39). 
Las estructuras de autoridad se distinguen en razón de si son "estructuras de toma de decisiones o de aplicación de estas últimas. En ambos casos, son las normas formales (Constitución, leyes, decretos, etcétera) las que determinan cuáles son esas estructuras de autoridad y las que regulan su ámbito competencial, quienes son las personas autorizadas para ocuparlas y bajo qué procedimientos, y cuáles son las funciones correspondientes a cada una de ellas" (idem). Sin embargo, esto no supone desconocer la importante influencia que las reglas de juego (informales) pueden ejercer sobre aquellos que ocupan posiciones de autoridad. Este estudio retoma, además, el término autoridades de implementación para referir a la capacidad institucional efectiva de que disponen los actores, en tanto estructuras de autoridad, para poner en marcha las metas estipuladas en el diseño institucional de una determinada política (Pressman y Wildavsky, 1998).

Para el análisis se recupera la visión propuesta por la primera generación de estudios sobre implementación de políticas públicas, representados en los análisis de Pressman y Wildavsky (1998) y en el marco teórico propuesto por la denominada tercera generación de estudios en implementación de políticas públicas (Mazmanian y Sabatier, 1981 cit. por Peters, 2006; Winter, 1990 cit. por Peters, 2006; O'Toole, 1990 cit. por Peters, 2006; Hill, 2001 cit. por Peters, 2006), que retoma la matriz analítica planteada en aquellos primeros estudios y define a la implementación desde una "lógica de gobernación" (Winter, 2006: 158 en Peters, 2006). Desde este modelo analítico, se define a la gobernación como "un régimen de leyes, reglas, decisiones judiciales, y prácticas administrativas que constriñen, prescriben y posibilitan, la provisión de bienes y servicios públicos" (idem). La implementación se define como un proceso; éste parte de una decisión política (el diseño de la política) que incluye la definición de metas y medios para alcanzarlas. Las políticas implican teorías. Se declaren éstas en forma explícita o no, las políticas apuntan a una cadena de causas que aparecen entre las condiciones esenciales y las consecuencias futuras. Las políticas se vuelven programas cuando, por un acto autorizado, se crean las condiciones esenciales. Los programas hacen que las teorías funcionen de manera que forjen el primer eslabón de la cadena causal que conecta las acciones con los objetivos. Así, la implementación es la capacidad de forjar eslabones subsecuentes de la cadena causal a fin de obtener los resultados que se desean (Pressman y Wildavsky, 1998).

La puesta en marcha de una decisión política conlleva una serie de decisiones subnacionales y acciones de distintos actores dirigidas a cumplir con el mandato de la autoridad federal. Además, durante el proceso de implementación política, las circunstancias cambian y las metas se alteran. Estos factores generan que el tránsito de una meta política (las condiciones esenciales) a su efectiva puesta en marcha no sea lineal. Antes bien, los actores encargados de la implementación se hacen responsables tanto de las condiciones esenciales como de los objetivos hacia los cuales se supone que aquellas conducen. En cuanto los actores ponen en funcionamiento las metas establecidas en el diseño institucional de una política pública, modelan, a partir de sus intereses y comportamientos racionales, la dirección y objetivos que adoptará la misma. Asimismo, transforman el juego de relaciones establecido con el entramado de actores encargado de definir su puesta en marcha.

Desde el enfoque de la gobernanza se aborda el modo específico en que se implementa la acción de gobierno, así como el entramado de actores públicos y privados que allí participan.

El sistema de educación superior se piensa como parte (o subsistema) del sistema político y se caracteriza por la función de producir; es decir, de transmitir decisiones vinculantes (Luhmann, 1991, 1993). Está compuesto por elementos 
correlacionados entre sí, definidos por determinados límites y en relación de interacción con el ambiente externo. ${ }^{1}$ El sistema de educación superior es estructuralmente "descentrado", es decir, no existe el predominio de una función sobre las otras ni coordinación entre ellas (Aguilar, 2007). En este marco, la implementación de sus políticas se define en términos de conflicto, negociación y/o alianzas, por parte de un entramado de actores: estatales, sociales no estatales, y paraestatales. ${ }^{2}$ En relación con la categoría "paraestatal", resulta conveniente especificar que representa a todas las autoridades académicas, estatales y no estatales, dado que todas (de diversa manera las autoridades de las instituciones "públicas" y "privadas") se diferencian del funcionario público típico, pero todas - al mismo tiempo- sufren restricciones, obligaciones impuestas por el Estado y un control estatal, que no permite confundirlos con actores sociales que, típicamente, actúan con un mayor grado de autonomía con respecto al Estado. ${ }^{3}$

La interacción entre éstos se caracteriza por el alto nivel de autonomía que separadamente pueden ejercer y por las relaciones de cooperación/conflicto expresados en términos de recursos de poder o capacidades institucionales (Cabrero, 2006; Acuña y Repetto, 2006) para el efectivo gobierno del sistema.

\section{Aspectos metodológicos}

Se trata de un estudio descriptivo-exploratorio. El objetivo es describir y analizar cómo se piensa al CPRES en el marco de la implementación de la Ley 24.521/95, qué modelo de gestión se esperaba instalar con su incorporación y cuál finalmente se puso en funcionamiento. Se abordó desde una perspectiva metodológica cualitativa. Para el análisis se integraron datos provenientes de dos tipos de fuentes: fuentes documentales de carácter normativo (leyes, decretos, resoluciones ministeriales y dictámenes) y documentación producida por el CPRES metropolitano (el conjunto de actas, resoluciones y acuerdos), y documentos de trabajo publicados por actores del ámbito académico en el campo de la educación superior. Además, se realizaron entrevistas en profundidad a actores clave del sistema educativo argentino. La guía de entrevista consideró las siguientes dimensiones: 1. Historia, 2. Funciones, 3. Agenda, 4. Actores y 5. Presupuesto.

\section{La elección del caso}

El estudio seleccionó el caso del CPRES región metropolitana; éste se compone de dos jurisdicciones, la ciudad de Buenos Aires y el Conurbano bonaerense. En la región metropolitana se concentra, en mayor medida, la actividad del sistema de educación superior. Se encuentran allí las universidades de mayor tamaño y tradición, como la Universidad de Buenos Aires (UBA) y la Universidad Nacional de La Plata (UNLP).

Durante los últimos años, en la región metropolitana se produjo una motorización del crecimiento institucional por parte del sector público

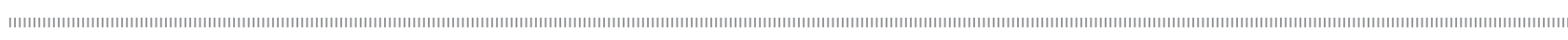

${ }^{1}$ El ambiente externo concentra subsistemas altamente diferenciados (economía, política, ciencia/tecnología, derecho, familia y vida privada, arte...) y autorreferidos, que interactúan entre sí siguiendo sus propios códigos de acción (en busca de recursos que sean procesados según su propia lógica de acción: objetivos, reglas, instrumental, imágenes de logro, etcétera).

${ }^{2}$ El sistema de educación superior está conformado por un intrincado entramado de actores. Se distinguen aquí tres categorías analíticas: a) los actores estatales, b) los actores sociales no estatales: los organismos internacionales, que si bien no tienen una territorialidad específica, capaz de categorizarlos como actores de una sociedad o un Estado determinado se presentan aquí, para efectos analíticos, como "actores sociales" no estatales (Repetto, 1997), y c) los actores paraestatales: integrado por el conjunto de rectores y/o autoridades de instituciones de educación superior universitarias y no universitarias, de gestión pública y privada, por asociaciones de rectores, grupos académicos y agrupaciones universitarias.

${ }^{3}$ La categoría paraestatal se adopta por la pertinencia y relevancia para significar a "todas las autoridades académicas, estatales y no estatales, dado que todas (de diversa manera los de las instituciones 'públicas' y los de las 'privadas') se diferencian del funcionario público típico, pero todas - al mismo tiempo - sufren restricciones, obligaciones impuestas por el Estado y un control estatal, que no permite confundirlos con actores sociales que, típicamente, actúan con un mayor grado de autonomía con respecto al Estado" (María Fernanda Potenza dal Masetto en entrevista, febrero 2011).
} 
y privado. Esto configuró un conglomerado institucional complejo. Desde el punto de vista territorial, la dinámica del sector privado tuvo lugar en la ciudad de Buenos Aires (CBA), mientras que el sector público adquirió mayor presencia en varios partidos del Conurbano bonaerense.

La CBA presenta un total de 24 universidades (dos nacionales y 22 privadas) y un total de 255 institutos de educación superior no universitaria (IESNU), de los cuales 49 son de gestión estatal y 206 de gestión privada (Krotsch y Atairo, 2008; Suasnábar y Rovelli, 2009; SPU, 2010).
El Conurbano bonaerense tiene 18 universidades (15 nacionales y tres privadas) y 204 IESNU, siendo 53 de gestión estatal y 151 de gestión privada (Krotsch y Atairo, 2008; Suasnábar y Rovelli, 2009; Secretaría de Políticas Universitarias (SPU), 2010).

En los últimos años el sistema de educación superior creció en términos cuantitativos pero no se modernizó (García de Fanelli, 2005). Esto ha tenido mayor visibilidad en el área metropolitana donde además se ha evidenciado con mayor claridad una tendencia institucional hacia nuevos modelos organizacionales (García de Fanelli, 2005 y 2009). ${ }^{4}$

Cuadro 1. Instituciones de educación superior en la región metropolitana, 2010

\begin{tabular}{|l|l|l|l|l|}
\hline Tipo de instituciones & Estatal & Privado & $\begin{array}{c}\text { Extranjeras- } \\
\text { internacionales }\end{array}$ & Total \\
\hline IESNU & 102 & 357 & 0 & 459 \\
\hline $\begin{array}{l}\text { Universidades e } \\
\text { institutos } \\
\text { universitarios }\end{array}$ & 17 & 25 & 2 & 44 \\
\hline Total & 119 & 382 & 2 & 503 \\
\hline
\end{tabular}

Fuente: elaboración propia con base en datos de SPU (2010) y Suasnábar y Rovelli (2009).

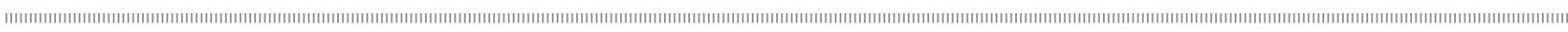

${ }^{4}$ La primera universidad argentina que se organizó por departamentos (antes que por facultades) fue la Universidad Nacional del Sur (UNS) en 1953. Un segundo momento de cambio organizacional se desarrolló a partir de los años noventa por un conjunto de universidades privadas. 


\section{Estructura del análisis y principales hallazgos \\ El entramado de actores}

En esta sección se presentan tres tipos de actores que conforman el contexto interinstitucional del CPRES.Un primer grupo de actores se encuentra en el ámbito nacional. Los actores estatales son: 1) el Ministerio de Educación, Ciencia y Tecnología de la Nación (MECyT), que interviene a través de la Secretaría de Políticas Universitarias (SPU); 2) la Comisión Nacional de Evaluación y Acreditación Universitaria (CONEAU), y 3) la Sindicatura General de la Nación (SIGEN), órgano rector de control interno en el ámbito gubernamental creado por la Ley 24.156/92 y encargado de supervisar el funcionamiento de las instituciones en el nivel ministerial.

En el nivel subnacional, se sitúan las autoridades públicas de los 24 gobiernos provinciales, encargadas de administrar el sector de educación superior no universitario.

Otro tipo de actores son los aquí denominados $p a-$ raestatales: 1) el Consejo Interuniversitario Nacional (CIN) integrado por los rectores o presidentes de las instituciones universitarias nacionales y provinciales reconocidas por la nación, 2) el Consejo de Rectores de Universidades Privadas (CRUP), 3) el Consejo Regional de Planificación de la Educación Superior (CPRES), organizado en siete regiones, que reúne en su interior a un representante de la SPU, a un representante de la máxima autoridad educativa provincial y a los rectores de las universidades públicas y privadas, de cada región, 4) el Consejo de Universidades (CU), precedido por un representante directivo del MECyT, y luego por el comité ejecutivo del CIN, la comisión directiva del CRUP un representante de cada CPRES, y un representante del Consejo Federal de Cultura y Educación. Finalmente, están los actores sociales no estatales: agrupaciones gremiales representadas en la Federación Nacional de Docentes Universitarios y representantes de distintos organismos internacionales.
La trama institucional está conformada por las interacciones entre el Estado y los actores que participan en el gobierno del sistema de educación superior. Está atravesada por intereses corporativos y voluntades particulares que en la resolución de sus tensiones van marcando las orientaciones que puede ir asumiendo la gobernanza territorial en los distintos contextos situacionales (Catenazzi et al., 2009).

\section{La gobernanza territorial: la creación del CPRES}

El origen del CPRES puede entenderse en el marco de un proceso de políticas orientadas al mejoramiento del sistema de educación superior y a la adecuación de sus programas académicos y científicos.

Surge en el marco de la Ley 24.521/95 de Educación Superior sancionada en 1995, luego de un proceso de fuertes debates en torno a la forma de concebir el sistema de enseñanza superior en la Argentina. En aquel escenario se identificaban dos concepciones en disputa sobre el modelo que debía adoptar el sistema de educación superior. Dichas posiciones se alineaban políticamente en dos grupos. Una de las posiciones nucleaba a los defensores de una mayor intervención del Estado en el sistema de educación o de un mayor regulacionismo estatal. Estaba conformado por funcionarios políticos, académicos y expertos de distintas disciplinas sociales, educativas, culturales y económicas. El modelo que proponían presentaba a la educación superior como un bien público y como recurso indispensable para el desarrollo integral de la nación. En ese marco, el Estado debía intervenir en la organización del sistema, para encarar políticas y programas educativos capaces de planificar, articular y organizar el conjunto de instituciones educativas y socioeducativas. Además, debía garantizar la igualdad y calidad de oportunidades para todos los habitantes del territorio frente al derecho a la educación. El Estado, para este grupo, se constituía como responsable de regular, asistir y focalizar los diferentes requerimientos 
de la oferta (universidades públicas e institutos de educación superior no universitaria) y de promover y asistir a la demanda de educación superior.

La otra posición reunía a los partidarios de una mayor autonomía (autonomistas) de las instituciones de educación superior y del retiro del Estado como promotor y agente de política activa para el sector. Se componía por una alianza de origen más heterogéneo pero con una fuerte unidad en sus acuerdos básicos. Estaba integrada por funcionarios políticos, académicos y expertos de disciplinas vinculadas a las ciencias económicas, así como por representantes de corporaciones empresariales nacionales y foráneas. Se sumaban a éstos los expertos provenientes de distintos organismos nacionales e internacionales que funcionaban como think tanks en la producción y formulación de políticas públicas y que adscribían al modelo socioproductivo propulsado por las reformas de primera y segunda generación ${ }^{5}$ implementadas por el gobierno de Carlos Menem entre fines de los ochenta y hasta mitad de los noventa. El modelo que proponían identificaba a la educación como un bien de mercado cuya calidad se garantizaba a través de la competencia y al que las demandas le asignaban pertinencia, y no la sociedad. Se pensaba que la regulación debía quedar en manos del mercado y el Estado nacional no debía intervenir como un actor "proveedor" de bienes.

Al promediar el año 1994, el entonces ministro de Educación Jorge Alberto Rodríguez, en representación del bloque autonomista, puso a consideración de la Comisión de Educación y Presupuesto de la Cámara de Diputados del Congreso Nacional el proyecto original sobre los Consejos de Planificación Universitaria Regional emanado del Poder Ejecutivo Nacional. El proyecto de ley llevaba por título "Régimen para el conjunto de las instituciones de educación superior y en particular para las universidades nacionales, privadas y provinciales" e inició su tratamiento en las sesiones ordinarias durante ese año. El mismo impulsaba una definición muy estructurada de las responsabilidades y funciones de los miembros de las instituciones de educación superior, la escasa (universidades públicas) o nula (universidades privadas) representación del personal docente, no docente y estudiantil en el gobierno universitario y la captación de recursos vía el mercado competitivo.

El grupo regulacionista con representación en el Congreso de la Nación percibió la propuesta como sumamente ortodoxa y generó una contrapropuesta centralmente interesada en suavizar la ortodoxia del texto original e inducir un cambio en el planteo sobre la regulación a cargo del mercado.

Luego de un proceso de intensos debates y complicadas negociaciones, se aprobó un marco regulatorio fuertemente normativo que satisfizo los objetivos de la posición autonomista e incluyó además las preferencias del grupo regulacionista. El proyecto original cambió su denominación convirtiéndose en la Ley de Educación Superior (LES) 24.521, promulgada en agosto de 1995. El texto de la Ley permite visualizar al CPRES como el arreglo institucional que surge de la transacción de las dos perspectivas. ${ }^{6}$ La contribución del sector regulacionista se produjo en el marco de un programa de "reforma de la educación y de la educación superior que fue parte de un proceso de carácter global cuyo núcleo ideológico residió en la dupla calidad-evaluación así como en una fuerte orientación al mercado. Al mismo tiempo que el Estado como eje de las transformaciones se reservó un papel regulador y evaluador" (Krotsch y Atairo, 2008: 27-28).

\footnotetext{
แกเแแแ

${ }^{5}$ De acuerdo con el uso que muchos documentos de los organismos internacionales ayudaron a generalizar, Camou (2002) distingue dos generaciones de reformas. La primera generación de reformas estaba centrada en la reestructuración de las relaciones entre Estado y mercado, en términos de una redefinición de las respectivas esferas de influencia. La segunda generación se ocupa - principalmente - de las mejoras en términos de calidad institucional, en particular, de aquellas áreas de política que son funciones indelegables del Estado (justicia, seguridad, educación, marcos regulatorios orientados a la competencia, etcétera).
} 
En este marco del debate se comprende la incorporación del CPRES como un instrumento de política pública, generando espacios para una mayor regulación ministerial en el sistema de educación superior. Sin embargo, en el nivel institucional, se profundizaba la autonomía de las universidades nacionales en su aspecto económico, autorizándolas a cobrar tasas y servicios (garantizando lo intangible de esos ingresos), a establecer los salarios del personal docente y no docente y facultándolas para dictar normas restrictivas al ingreso.

Entonces, el CPRES tiene el propósito inicial de generar espacios de planificación pero en un marco autonómico de laissez faire.

\section{El contexto legal}

El CPRES se piensa como un instrumento de política activa a cargo del Estado nacional. Su diseño institucional encarna el espíritu general de la Ley 24.521/95 caracterizada por un enfoque de planificación descentralizada y regional, con alto nivel de autorregulación del sistema.

En ese contexto, el CPRES se propone como uno de los mecanismos interinstitucionales de coordinación sistémica (educación superior como un conjunto mayor que educación universitaria). Esto es, asignándole la concertación integral del sistema de educación superior que comprende a dos subsistemas: el de educación universitaria (pública y privada) y el de educación superior no universitaria (institutos de enseñanza terciaria, formación docente y técnica).

Además, la Ley prevé que el CPRES desarrolle múltiples objetivos: a) la articulación entre el nivel medio y el superior; b) la articulación entre el nivel universitario y no universitario, c) la detección de necesidades de educación superior a nivel regional, posibles de atender por el marco institucional y, por último, d) la coordinación entre Universidades.

En los artículos 10, 71 y 72 contiene referencias específicas. Según el artículo 10, "la articulación a nivel regional estará a cargo de los Consejos Regionales de Planificación de la Educación Superior, integrados por representantes de las instituciones universitarias y de los gobiernos provinciales de cada región".

El artículo 71 los incluye dentro del conjunto de órganos de coordinación y consulta, expresando que "serán órganos de coordinación y consulta del sistema universitario, en sus respectivos ámbitos, el Consejo de Universidades, el Consejo Interuniversitario Nacional, el Consejo de Rectores de Universidades Privadas y los Consejos Regionales de Planificación de la Educación Superior".

El artículo 72 señala la participación de un representante de cada CPRES — que deberá ser rector de una institución universitaria- en la conformación funcional del Consejo de Universidades.

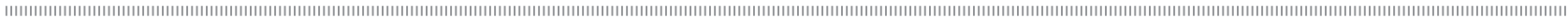

${ }^{6}$ A partir de las consideraciones señaladas por Juan Carlos del Bello en una lectura preliminar de este trabajo, considero apropiado precisar el alcance analítico e interpretativo que aquí se hace de las categorías "autonomista" y "regulacionista". Éstas se han utilizado en estudios previos (Camou, 2005, 2007, 2001; Krotsch, 2001, 2007, 2008; Pérez Rasetti, 2007, 2010), y aquí se recuperan para marcar las diferencias observadas entre dos posiciones políticas. La posición autonomista caracterizada por propiciar una mínima intervención estatal en el ámbito público. La intervención del Estado se justifica en la medida que asegura las reglas de juego dentro de las que operan los individuos.

La posición regulacionista visualiza al Estado como un actor central. No sólo garantiza las reglas de juego sino que tiene un rol activo y conductor para asegurar la gobernabilidad del sistema. Por tanto, sostiene un mayor intervencionismo estatal. De la transacción entre estas dos posturas aparece lo que Del Bello (2010) denomina "neo-regulacionismo". Desde la perspectiva analítica que guía el trabajo propuesto, se entiende que el carácter "neo-regulacionista" resulta del sistema de acuerdos y arreglos políticoinstitucionales generados en torno al debate para sancionar la LES. Esto supone reconocer dos grupos de actores con posiciones de poder diferentes y, por tanto, con intereses diversos. De manera que, visto de este modo, no es posible definir el carácter de "neo-regulacionista" como un atributo general o unívoco. Asimismo, se reconoce que luego de la sanción y puesta en marcha de la LES, esos dos grupos comenzaron a confundirse en una misma línea político-ideológica, de difícil definición, y asumieron en grado creciente la necesidad de implementar políticas caracterizadas por un nuevo tipo de regulaciones o "neo-regulacionistas".
} 
La obtención de dicho marco regulatorio expresó, por un lado, los intereses contenidos en la propuesta de la posición autonomista que, desde una perspectiva política, buscó mejorar los problemas de calidad educativa y eficiencia institucional, generados por una anterior desatención y restricción presupuestaria, la ineficacia en los métodos de planificación centralizada y la falta de integración sistémica. Pero además representó para la posición regulacionista la oportunidad de incorporar, desde una mirada técnica, los objetivos de planificación descentralizada de nivel regional.

En ese marco, la decisión de crear los CPRES estuvo motorizada por razones técnico-políticas vinculadas a la necesidad de fortalecer la capacidad organizativa de "territorios" para el desarrollo de sistemas sociales, culturales y productivos locales, configurando una red de instituciones de educación, institutos de investigación y empresas conectadas con el tejido social e institucional de cada localidad o región.

De esa manera se recuperaba la tradición de abordaje regional que desde los años sesenta, y aun en los periodos dictatoriales, otorgaba al Estado un papel activo en la planificación e integración del sistema como una forma superadora de la división provincial. El CONADE primero, luego la Secretaría de planificación a nivel de la presidencia, la regionalización de instituciones como el Consejo Nacional de Investigaciones Científicas y Técnicas (CONICET) o el Instituto Nacional de Tecnología Agropecuaria (INTA), son ilustrativos de este enfoque. Incluso el Plan TAQUINI (H) obedecía a un criterio de regionalización.

Este paradigma tuvo un primer antecedente en la legislación argentina con la última ley de la democracia de 1974 (Ley 20.654), conocida como Ley Taiana. E1 artículo 52 preveía diversas instancias de planificación. Incluso mediante Decreto 451 del 5 de julio de 1973 se suspendía la creación de nuevas universidades, tanto estatales como privadas, hasta tanto tuviera lugar un proceso de planificación, norma que estuvo vigente hasta fines de la década de los ochenta.

La configuración regional del CPRES en parte parece ser tributaria del paradigma de planificación de los sesenta: el Noroeste Argentino (NOA), el Noreste Argentino (NEA) y la Patagonia. Resulta heterodoxa a esa tradición el armado de los CPRES , Centro Oeste (COES) y Centro Este (CEE), cuando tradicionalmente las regiones fueron Cuyo, Centro y Mesopotamia. ${ }^{7}$

La Ley de Educación Superior (LES) no especificó funciones para el CPRES pero sí lo hizo la Resolución Ministerial $602 / 95,{ }^{8}$ por medio de la cual se reglamentó el contenido de la LES y se asignó al CPRES funciones y mecanismos específicos de planificación, coordinación y consulta del sistema, con el objetivo básico de promover la integración de las instituciones de educación superior en el contexto regional, así como el intercambio y la reflexión conjunta entre dichas instituciones, los representantes de los estados provinciales, las instituciones sociales intermedias y los sectores productivos de la región.

Sin embargo, el momento de reglamentación de las funciones del CPRES pareció renovar la tensión entre los funcionarios políticos de la posición autonomista, responsables de la puesta en marcha de dicha norma y los representantes del grupo regulacionista, sin identidad partidaria en el Poder Ejecutivo Nacional.

Es evidente que la reglamentación de los artículos 10,71 y 72 contenidos en la LES se concretó parcialmente con la Resolución Ministerial 602/95 y se completó con la ejecución de dos

\footnotetext{
Н| -

${ }^{7}$ La región bonaerense en los años sesenta se definía como pampeana, y alcanzaba a la provincia de la Pampa. Hacia 1983 , con el retorno a la democracia se crea la región Patagónica y se incorpora esta provincia al "Sur Argentino".

${ }^{8}$ www.me.gov.ar/spu/guia_tematica/CPRES/cpres-normativas.htm
} 
decretos nacionales, en conformidad con lo dispuesto por el artículo 99, inciso 2, de la Constitución Nacional Argentina.

E1 22 de septiembre de 1995 el artículo 11 del Decreto Nacional 499 estableció que "en la reglamentación que el Ministerio de Educación y Cultura dicte para la organización y funcionamiento de los CPRES, deberá preverse el procedimiento de elección de los representantes de esos cuerpos ante el Consejo de Universidades, así como la duración de sus mandatos".

Posteriormente, el 4 de junio de 1996 se publicó en el Boletín Oficial el Decreto Nacional $576 / 96$, que reglamentó las consideraciones específicas de los artículos 10, 71 y 72 de la LES pero sólo en referencia a la autorización, creación y modificación en los estatutos y planes de estudios de las IES con el propósito de restringir la expansión territorial del sistema. En 1999 este Decreto fue derogado junto a un Decreto anterior (el 2282/93) y se emite el Decreto 1047/99, que define al CPRES como un organismo de consulta no vinculante para evaluar la creación de nuevas sedes, subsedes y extensiones áulicas fuera del CPRES de pertenencia.

En resumen, si bien la Ley 24.521 recupera para el CPRES el tipo de abordaje regional propuesto por el paradigma de planificación iniciado en los años sesenta, la implementación de sus objetivos y mecanismos es un proceso que aún se encuentra en marcha. El CPRES ha logrado estructurase como un espacio de autoridad, en tanto actor estatal, en el gobierno del sistema de educación superior. Pero debido a las tensiones de la que es tributaria la normativa no ha podido pasar a la acción pública.

\section{Estructuras de autoridad.}

\section{Las funciones efectivas del CPRES}

Hasta aquí se ha relatado el proceso que guió la conformación de un marco regulatorio para el CPRES. En esta sección se analizará el modelo de planificación que efectivamente funciona.

Para ordenar el análisis de los roles que el CPRES ha puesto en funcionamiento desde 1996 se distingue la sucesión de tres periodos políticos. El primero se extiende de marzo de 1996 a diciembre de 1999. En un sentido amplio, este periodo se caracteriza por la consolidación de la estructura organizativa de la SPU, creada en 1993. La SPU se organizó en dos subsecretarías: la de Coordinación Universitaria y la de Programación, y tres direcciones: Gestión Universitaria (ex Asuntos Universitarios), Economía e Información y Dirección Nacional General, en la que se integraban los distintos proyectos ligados al Programa de Reforma de la Educación Superior (PRES) (Krotsch y Atairo, 2008). ${ }^{9}$ Su creación está en estrecha vinculación con la creciente expansión y diversificación institucional de las últimas décadas. Esto supuso un incremento importante en las actividades de política universitaria, así como del cuerpo técnico y administrativo encargado de su implementación y seguimiento. En este proceso de ampliación e institucionalización de las actividades de regulación también jugó un papel importante la formalización de llamados a concurso para los distintos cargos de la estructura, lo que implicó un incremento cuantitativo y cualitativo de la capacidad técnico-burocrática del organismo, así como de su importancia dentro del Ministerio de Educación de la Nación (Krotsch y Atairo, 2008).

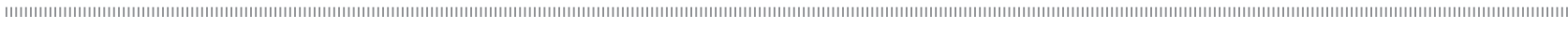

${ }^{9}$ En 1995 se pone en marcha el PRES, cuyo objetivo fue el mejoramiento de la eficiencia y calidad de la gestión universitaria y también de la reforma académica y administrativa. El Programa tenía varios componentes que incluían: el fortalecimiento institucional del sector universitario a través de la CONEAU, el SIU (Sistema de Información Universitaria), el Sistema de Información y Asignaciones Presupuestarias de Recursos y el FOMEC (Fondo para el Mejoramiento de la Capacidad), así como el Programa de Gestión y Planificación de la propia SPU (Krotsch y Atario, 2008).
} 
El segundo periodo se ubica entre diciembre de 1999 y principios de 2002. Se caracteriza por el predominio de un contexto de restricción presupuestaria en el nivel nacional. En particular por la reformulación del diseño institucional que dio origen al CPRES.

Después, los cambios de signo y conducción política en la Presidencia de la Nación, - primero con Eduardo Duhalde (2002-2003) y luego con Néstor Kirchner (2003-2007)—, determinan el tercer periodo del análisis que llega a la actualidad. Éste podría caracterizarse por una mayor intervención estatal en el funcionamiento del sistema de educación superior y por un incremento $\mathrm{y}$ fortalecimiento de las funciones ejecutivas que despliega el CPRES en relación con las actividades de tutela previas. Además, entre 2003 y 2007, se registra una mayor participación dentro del ámbito CPRES de los rectores de las universidades públicas y privadas. Si bien esta participación es uno de los elementos constitutivos del CPRES como espacio público de consenso y negociación, se fortaleció luego del periodo de reforma institucional del CPRES de los años 1999-2002. El interés de estos actores sociales se orientó a la recomposición de la estructura organizativa del CPRES y no sólo a su funcionamiento. Dicha participación es notable en la concurrencia de rectores y secretarios académicos a las dos reuniones plenarias (de carácter anual) y en el apoyo económico brindado por las universidades nacionales y privadas, sea proporcionando infraestructura edilicia o asignando recursos para movilidad.

Para comenzar, es posible observar en estos tres periodos que tanto las condiciones de contexto como la intervención de otros actores han interpelado la figura del CPRES. Ambos factores impulsaron la intromisión progresiva del CPRES en la acción pública y dieron lugar a una mayor especificación de los roles y funciones puestos en marcha.

\section{Primer periodo: marzo de 1996 a diciembre de 1999}

El CPRES se puso en funcionamiento a mediados de 1996 en el ámbito de la Subsecretaría de Coordinación Universitaria de la SPU. A partir de ese año se organizó para desarrollar la función de regulación de la oferta de actividad académica en el nivel nacional. Esa función no se cumplió de forma sistemática sino que su intervención se orientó a evaluar la oferta académica de cada región, en función de los pedidos de creación de sedes, subsedes y extensiones áulicas por parte de las instituciones de educación superior pública y privada.

En el nivel nacional, el crecimiento o expansión del sistema de educación superior se efectúo de dos maneras. Por un lado, a través del proceso formal que dio lugar a la creación de instituciones universitarias públicas y privadas. ${ }^{10} \mathrm{~A}$ la vez, entre los años 1997 y 1999, se registró un aumento desorganizado de sedes, subsedes y extensiones áulicas de instituciones de educación superior ya existentes, para el dictado de cursos o carreras presenciales y/o a distancia. Estas nuevas creaciones no se efectuaron de acuerdo con el reglamento oficial, esto es, a través de una previa consulta al CPRES, sino mediante mecanismos institucionales informales que dejaron fuera del alcance del CPRES la regulación de esas actividades académicas. Entonces, se observó su dificultad para asumir la tarea de regulación de la oferta en el nivel nacional, lo que generó una falta de visibilidad oficial para el desempeño efectivo de la acción pública.

Además, el desempeño de esta función se vio limitada por las condiciones de restricción presupuestaria generadas por el contexto de crisis política y socioeconómica que afectaba a los sistemas políticos de la región en general y al sector educativo en particular.

\footnotetext{
${ }^{10}$ Las leyes y normas que regulan la creación de IES son: a) la Constitución Argentina de 1853, reformada en 1994, b) la Ley de Educación Superior (LES) de 1995, y c) el Decreto Nacional 576/96.
} 
Para el caso argentino, entre el bienio 1997-98 el modelo neoliberal (definido por la separación entre Estado y mercado, fronteras "para adentro", por la inserción competitiva en un mercado global, fronteras "hacia afuera", y por la minimización del Estado) ${ }^{11}$, plasmado en el Plan de Convertibilidad $^{12}$ lanzado en abril de 1991, comenzó a evidenciar una serie de dificultades en sus postulados básicos. Se replantearon las condiciones de interacción funcional entre el Estado y el mercado externo. ${ }^{13}$ Esto determinó un contexto de escasez de recursos públicos y, en particular, obstaculizó la intervención ministerial a través del CPRES para ejercer una efectiva regulación de la oferta académica por región.

En términos económicos, la proyección presupuestaria del Ministerio de Cultura y Educación de la Nación fijó para 1997 una estructura presupuestaria fragmentada en diversos programas por un total de 148800000 pesos. En 1998 se estableció una suma total de 120600514 pesos, ${ }^{14}$ lo que implicó una reducción de 28199486 en relación con el periodo anterior.
Por otro lado, en octubre de 1997 se celebraron elecciones legislativas. El gobierno de Menem fue derrotado. Una alianza integrada por la Unión Cívica Radical (UCR) y el Frente País Solidario (FREPASO) logró reunir casi el $46 \%$ de los votos contra el $36 \%$ de las preferencias justicialistas. Se produjo un fortalecimiento en el nivel parlamentario del Partido de la Alianza y una ruptura del Partido Justicialista en dos sectores políticos diferenciados: el menemismo por un lado, y otro grupo encabezado por Eduardo Duhalde. Este acontecimiento abrió entonces un doble proceso de descomposición. A la descomposición del modelo neoliberal le siguió la ruptura de la matriz política que lo sostuvo.

Sin embargo, es posible determinar otros factores que entre los años 1996 y 2001 impulsaron un mayor involucramiento del CPRES en tanto estructuras de autoridad con funciones específicas de regulación.

Uno de ellos se inició con el planteo en torno a la necesidad de instalar un nuevo modelo de financiamiento para el sistema de educación superior, lo que conllevó un fuerte cuestionamiento al diseño

\footnotetext{
${ }^{11}$ La definición puede encontrarse en Camou, 2002.

12 "E1 $1^{\circ}$ de abril se estableció la convertibilidad, sancionada por el Congreso Nacional a través de la ley 23.928, que fijó como paridad cambiaria sin límite temporal la de 10000 australes por dólar. La ley dispuso la libre convertibilidad de la moneda nacional con respecto a cualquier moneda extranjera, y estableció que los contratos deberían ser cumplidos en la moneda que fijaran las partes. Se prohibió la emisión de dinero no respaldado en un $100 \%$ por reservas de libre disponibilidad (oro, divisas, títulos de otros países y títulos nacionales emitidos en moneda extranjera) y se anularon las indexaciones, intentando evitar la traslación de la inflación pasada hacia el futuro. El Banco Central fue autorizado también a cambiar la denominación del signo monetario. En consecuencia, a partir del $1^{\circ}$ de enero de 1992 se estableció el peso como moneda de curso legal, equivalente a 10 000 australes, y se fijó el tipo de cambio de 1 peso por dólar..." (Rapoport, 2000: 976-977).

${ }^{13}$ De acuerdo con Rapoport: "El esquema de convertibilidad dependía de un persistente ingreso de oro o divisas del exterior, ya sea a través del superávit de la balanza comercial o de flujos positivos de capitales..." (Rapoport, 2000: 975).

${ }^{14}$ El Presupuesto Nacional (Ley 24.764/96) de 1997 asignó al Ministerio de Educación y Cultura de la Nación la suma de \$111 800000 con el objetivo de mejorar la calidad de la enseñanza superior y alcanzar una mayor eficiencia del sistema universitario. Además, se dispuso un total de \$37000 000 distribuidos así: a) \$2 800000 para el sistema de información universitaria, b) \$1 400000 para la red de información universitaria, y c) \$32 800000 para la ejecución de las transferencias a las universidades nacionales incluidas en el Programa de Mejora de la Calidad (POMEC). En 1998, el esquema presupuestario (Ley 24.938/97) fijó como crédito a distribuir por el Ministerio de Cultura y Educación un total de \$23 600514 para los Programas de Apoyo a la Gestión y a la Calidad universitaria. La distribución de dicho monto se estableció del siguiente modo: a) 14000000 pesos para Apoyo al crecimiento institucional, b) $\$ 7000000$ para el Mejoramiento de la Calidad de las universidades nacionales, c) $\$ 2600$ 514 para la atención de eventuales emergencias de las universidades nacionales. También se asignaron créditos directos al Ministerio de Educación y Cultura de la Nación para el desarrollo de distintos programas de mejoramiento del sistema universitario. Se destinaron $\$ 70000000$ al Programa de incentivos a los docentes investigadores. Y otros \$27000 000 para otros Programas de Desarrollo Universitario: a) \$7000 000 para el Programa de Financiamiento a la Enseñanza Universitaria (PROFIDE), b) \$15 000000 para el Programa de Financiamiento de Inversiones, c) \$5 000000 para el Programa de Apoyo a las Universidades Nacionales nuevas.
} 
formal de la SPU en relación con sus políticas de planificación y regulación de la oferta educativa.

Hacia 1998 se estableció un debate entre determinados actores con posiciones diferentes en relación con la diversificación y redistribución de los recursos en las instituciones. Los funcionarios públicos del sector más liberal del peronismo pensaron que los requerimientos del gasto en educación superior, ciencia y tecnología competían con otras prioridades de gasto social y educativo visualizados como más apremiantes. Entonces, propusieron un cambio de enfoque en la distribución del gasto público, más concentrado en la necesidad de los estudiantes, en tanto "consumidores", que en los requerimientos de las instituciones. Asimismo, impulsaron la creación de distintos mecanismos de arancelamiento de la educación pública. En oposición a esta propuesta se reunió un núcleo de actores conformado por un sector del campo universitario, los gremios docentes representados en CONADU y FEDUN ${ }^{15}$ y distintas agrupaciones estudiantiles. Este grupo de actores sociales bregó por el predominio público en la gestión y distribución del gasto del sistema de educación superior.

La intensidad de esa disputa desvío la atención ministerial para encarar el fortalecimiento de las instancias de planificación, coordinación y regulación educativa, pero estimuló la participación pública de la Secretaría Ejecutiva del CPRES para sostener el diseño formal de la SPU y negociar en ese marco una mejora en el esquema presupuestario con el propósito de fortalecer su capacidad técnico-operativa.

En concomitancia, entre 1998 y 2002, se evidencia la interpelación de dos agencias estatales al accionar público del CPRES. Primero, por parte de la CONEAU que expresó, a la vez, las demandas del CU y el CIN. En 2002 por la SIGEN en tanto agencia gubernamental de control interno del Poder Ejecutivo Nacional (PEN).
En 1998, la CONEAU emitió una declaración que reveló el crecimiento de sedes, subsedes y extensiones áulicas y las distorsiones que implicaba respecto de las instancias de planificación.

Por su parte, los rectores de las universidades nacionales más antiguas y de mayor tamaño se preocuparon por esta expansión y a través de una propuesta de regulación retomaron el tema planteado por la CONEAU.

En el marco de un contexto de escasez presupuestaria, la preocupación de aquellos rectores nacionales se orientó, por un lado, a limitar la creación de nuevas IES con la intención de resguardar el esquema de distribución presupuestaria. Pero además expresó el interés por definir su participación como órgano de regulación y consulta vinculante del sistema para atender los requerimientos de creación de nuevas sedes, subsedes y extensiones áulicas. Dicha propuesta se acordó en el CU el 24 de junio de 1999, por medio del Acuerdo Plenario número 10 y se elevó al PEN.

Podría decirse que desde esa fecha hasta 2005 se discutió entre el CU y el CPRES la estructura de autoridad que debía adoptarse para la efectiva regulación de la oferta académica. En esa oportunidad, el PEN ratificó la figura del CPRES, a través del Decreto 1047 del 23 de septiembre de 1999, como organismo regulador de la oferta académica del sistema, aun cuando incorporase la opinión del CU para el desempeño de la misma.

\section{Segundo periodo: diciembre 1999 a principios de 2003}

Hacia fines de 1999, Argentina experimentaba la profundización de la crisis del modelo neoliberal de carácter mundial. Los problemas se evidenciaron hacia 1998 cuando al estrecharse el flujo financiero externo se inició un largo ciclo recesivo, que habría de durar al menos cinco años (Romero, 2003).

${ }^{15}$ Comisión Nacional de Docentes Universitarios (CONADU) y Federación de Docentes Universitarios (FEDUN), respectivamente. 
En medio de la recesión hubo elecciones presidenciales y el Partido de la Alianza, que reunió a los principales opositores del Partido Peronista, ganó con cierta holgura (Romero, 2003: 107).

El programa de gobierno de la Alianza destacó la necesidad de conformar una administración transparente y racional sin abandonar los principios rectores del modelo neoliberal. En ese sentido, anuló el ámbito del CPRES como espacio de autoridad y se reformuló la estructura de la SPU con la fusión de las subsecretarías de Coordinación Universitaria y Programación. Con ello, se eliminó la dirección del CPRES y a partir de allí dejaron de tener misión específica y recursos asignados permanentemente. Dicho proceso de reformulación se acompañó de una modificación presupuestaria que redujo los recursos humanos del CPRES de diez (un secretario ejecutivo, siete técnicos, y dos administrativos) a dos empleados (un subsecretario y un administrativo).

Otra dificultad resultó ser la inestabilidad política generada en marzo de 2001 tras el anuncio del entonces ministro de Economía, Ricardo López Murphy ${ }^{16}$ de ajuste presupuestario para el área educativa. Como resultado de ese anuncio, el ministro de Educación Hugo Juri y su equipo presentaron la renuncia. Después, se desató una fuerte crisis políticoinstitucional que impidió al CPRES encarar cualquier proceso de mejora y/o reforma. Dicha crisis finalizó el 21 de diciembre con la dimisión del presidente de la nación, Fernando De la Rúa. ${ }^{17}$

En 2002, un informe de la SIGEN marcaba que el CPRES no había contado con una política de planificación capaz de establecer las direcciones en materia de oferta educativa definida para cada región en particular. Además, no se lo había provisto de un registro formal de universidades, sedes y convenios de articulación ya existentes en la región, ni había formulado controles sobre los convenios de articulación que desde 1999 realizaban las universidades con los institutos de enseñanza terciaria.

Aquel informe pretendía superar la discusión política de carácter bipolar en torno al modelo de financiamiento público-privado, para demostrar a las autoridades del Ministerio de Educación y en particular a la Secretaría Ejecutiva del CPRES que desde1996 hasta 2002 no se habían alcanzado los objetivos de acción pública para los que fueron creados.

\section{Tercer periodo: desde 2003 a la actualidad}

En 2003 asume la conducción de la Secretaría Ejecutiva del CPRES en la SPU uno de los representantes del grupo que integró la corriente de regulacionismo estatal en la década de los noventa. Inmediatamente, se identificó la necesidad de realizar un diagnóstico oficial sobre las funciones desarrolladas por los CPRES en el marco del proceso de implementación de la LES. El diagnóstico hacía referencia a una resignificación de los objetivos que motivaron la creación del CPRES y la intención de retomar los fundamentos de la posición original. Con base en este diagnóstico se propusieron reimpulsar las funciones de planificación y coordinación integral del sistema de educación superior. En ese sentido, una de las primeras acciones implementadas fue asignar al CPRES un conjunto de líneas de política pública

\footnotetext{
${ }^{16}$ Datos obtenidos del estudio realizado por Diego Santori (2005).

${ }^{17}$ El historiador Luis Alberto Romero presenta una forma de apreciar la reciente crisis del sistema político argentino: "El cataclismo se produjo a fines de 2001. Primero, una fenomenal corrida bancaria, secuela de la retirada presurosa de las inversiones financieras, llevó a una congelación de todos los depósitos - el corralito-y consecuentemente a una crisis económica vertiginosa, acentuada más tarde por la devaluación asimétrica, que dejó un problema entre deudores y acreedores insoluble en términos lógicos. Paralelamente, las protestas sociales - algunas espontaneas, otras movidas por los aparatos partidarios peronistas- y finalmente la crisis política desencadenada por los gobernadores peronistas, provocaron la renuncia del presidente, institucionalmente agravada por la renuncia, un año antes, del vicepresidente" (Romero, 2003: 109).
} 
orientadas a mejorar la articulación, coordinación y planificación del sistema.

En ese marco, una primera línea de política pública plantea al CPRES como responsable de la implementación del programa de articulación entre carreras e instituciones del nivel superior. Además, en 2004 se pone en marcha un programa de articulación entre universidades y escuelas medias. El principal objetivo de este programa consiste en promover acciones coordinadas entre las universidades y las instituciones educativas provinciales, tendientes al mejoramiento de la calidad de la escuela media y a contribuir al pasaje de los jóvenes al nivel superior, facilitando el acceso y permanencia de los estudiantes en los primeros años de la universidad. Con la intención de ampliar y profundizar este trabajo, se convocó al conjunto de universidades nacionales a participar de dicho programa con la presentación de un proyecto institucional. A partir de esto se crea un Mapa de la Oferta de Educación Superior y se aprueba e implementa el Registro de Convenios de Articulación y Asociación y de Experiencias de Articulación para ordenar la información sobre articulación y cooperación entre instituciones del sistema que tuvieran por objeto la implementación total o parcial de carreras universitarias.

Entre 2005 y 2006 es posible visualizar el desarrollo y sistematización de distintas experiencias de articulación entre instituciones universitarias y no universitarias así como también entre los dos últimos años del nivel educativo medio y el primer ciclo del nivel superior. ${ }^{18}$

En 2007 se definen los ejes de calidad para nuevas ofertas académicas en el ámbito de competencias del CPRES y se crea la Comisión de Evaluación Permanente. Además, se establecen un conjunto de recomendaciones o de estándares para que la apertura de sedes, subsedes y extensiones áulicas tenga una calidad equivalente. En la actualidad, la Comisión de Evaluación Permanente es una de las principales áreas de trabajo del CPRES. Sus miembros se reúnen a pedido de las universidades interesadas en la apertura de una sede, subsede o extensión áulica y evalúan la actividad académica que desarrolla la región. Sin embargo, las tareas de esta Comisión introducen un factor de tensión con las funciones que desarrolla la CONEAU porque, de acuerdo con las normas referidas al CPRES, no le correspondería fijar políticas de calidad.

Hacia fines de 2007 se crea la Comisión de Formación Docente. Esta Comisión habría surgido a pedido de las universidades nacionales del CPRES metropolitano para conocer la actividad desarrollada por el Instituto Nacional de Formación Docente (INFOD) cuya reciente creación (Ley 26.206/06) les interesaba particularmente. La emergencia del INFOD habría despertado algunos temores en las universidades vinculados al rol del Instituto como un organismo que atentaría contra la autonomía de las universidades estableciendo curricula homogéneos en todo el país para la formación docente y disminuiría el reconocimiento de nomencladores de los títulos de docencia que tienen las universidades. Esto abría un foco de tensión entre las universidades (de gestión nacional) y las autoridades educativas de cada provincia, porque las universidades visualizaron en el INFOD a un actor que venía a hacer fuerza del lado de las provincias y en contra de las universidades. En ese contexto, la Comisión de Formación Docente incorpora dentro de las reuniones plenarias a dos nuevos actores: un representante del INFOD y uno del Instituto Nacional de Enseñanza Tecnológica (INET). De este modo, la presencia de éstos se vuelve significativa para la configuración de relaciones con las universidades. Sin embargo, aún no logran una titularidad legal en el CPRES.

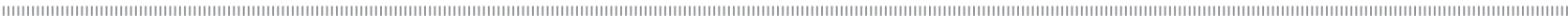

${ }^{18}$ El registro de experiencias puede encontrarse en línea: www.cpres.siu.edu.ar/ 
El trabajo del CPRES se organiza a través de una agenda que establece su secretaría ejecutiva y, en algunos casos, a partir de los aportes que hacen los rectores o sus representantes en los plenarios de cada CPRES.

Las cuestiones sobre las cuales interviene efectivamente se deciden en acuerdo con los miembros del pleno de cada CPRES, pero en la mayoría de los casos tiene la iniciativa la Secretaría Ejecutiva presentando al pleno de cada CPRES los temas por discutir.

La sesión plenaria de cada CPRES está integrada por representantes de las universidades públicas y privadas con sede en la región, por las autoridades educativas provinciales y por un representante de la Secretaría Ejecutiva del CPRES. Además participan como miembros no titulares del CPRES un representante del INFOD y de INET. La participación de cada actor en las reuniones plenarias es variable. No hay representación importante de las provincias. Esto se comprendería como una baja internalización, a nivel subnacional, de lo que significa el espacio CPRES como instancia de gobierno. También se explica por la insuficiencia presupuestaria de las instituciones educativas responsables de los gastos de viáticos.

Se evidencia, en cambio, una participación mayor de la educación superior privada. La particularidad del área metropolitana es que la mayor parte de las universidades nacionales, con excepción de la UBA, está en el conurbano. Además, las universidades nacionales que concentra el conurbano pertenecen a una región que geográfica y socialmente configura una especie de bloque propio y tienen una dinámica de relación entre ellas muy fuerte. Esto se evidencia en la creación de la red de universidades del conurbano que, en un sentido amplio, funciona como una especie de CPRES dentro del CPRES. En cambio, las universidades privadas tienen una lógica de actuar por su propia cuenta. No obstante, ambas asisten regularmente a las reuniones plenarias, con intención cooperante.

\section{Conclusiones}

Las conclusiones que arroja este estudio dan cuenta de que el CPRES se creó como un organismo de nivel intermedio con capacidad jurídico-institucional para ejercer la coordinación y regulación sistémica de la educación superior argentina. Sin embargo, esta meta inicial, señalada en el diseño institucional de la política que da origen al CPRES, se fue alterando en el curso de su implementación. En ese marco pueden identificarse tres elementos clave:

Primero, la influencia del contexto o entorno intrainstitucional definido por la interacción y diferenciación funcional del entramado de actores que componen el denominado sistema de educación superior. La interacción se ha expresado en términos de cooperación y/o conflicto entre los miembros de la comunidad universitaria: las universidades nacionales y las privadas, los gobiernos provinciales como representantes de los institutos de educación superior no universitaria y el rol del MECyT. Además, la interpelación de otros actores como el CU, la CONEAU y la SIGEN, definieron fuertes demandas de funcionamiento a través de recomendaciones y declaraciones oficiales.

Segundo, las características propias de la estructura institucional de cada organización, de fuerte base burocrática, dificultaron el ejercicio de coordinación y articulación entre los actores en el curso de la implementación. Al decir de Pressman y Wildavsky (1998: 228):

El hecho de ordenar un organismo o persona que coordine no le dice a ésta lo que tiene que hacer, así que no sabe si debe presionar o negociar, ejercer su autoridad o asegurar un consentimiento. Tenemos así un aspecto de un rasgo, en apariencia conveniente, de una administración antiburocrática que encubre los mismos problemas - pugna versus cooperación, coerción versus consentimiento- cuya invocación se supone que resuelve. 
Finalmente, debido a la influencia del contexto interinstitucional. Es decir, por la escasa interacción y baja vinculación generada entre el sistema científico tecnológico de las principales universidades nacionales y el subsistema económico y social.

En definitiva, se intentó dar cuenta de que, en términos legales, el CPRES se pensó como un ámbito de planificación y coordinación de la educación superior en el nivel regional. Sin embargo, su puesta en funcionamiento ha mostrado al CPRES como un organismo con capacidades institucionales muy limitadas (carencia de una estructura presupuestaria básica, recursos económicos y administrativos) para negociar intereses y efectivizar acciones concretas de regulación de acuerdo con lo que su normativa indica. Asimismo, se ha mostrado como un organismo muy sensible a la definición de cambiantes metas políticas gubernamentales. En efecto, el diseño institucional que instituye al CPRES, responde más a una lógica de centralización del poder en el gobierno nacional que a una verdadera descentralización con efectiva intervención de las jurisdicciones provinciales. Esto ocurre en un contexto de descentralización política y financiera del sistema educativo hacia los gobiernos subnacionales.

Pero a la vez, las instituciones universitarias identificaron en el CPRES un espacio conveniente de reunión y negociación. El grado variable de participación de las universidades en los tres periodos da cuenta de una intención cooperante para mejorar y revitalizar su funcionamiento. En ese marco el logro más importante del CPRES es su reconocimiento como un organismo de regulación que formalmente reúne al conjunto diverso de actores en un mismo espacio de diálogo. Este espacio se instituye como un espacio de poder y negociación en el que se dirimen intereses, establecen prioridades de acción y se formalizan los conflictos. En términos políticos, ha limitado la agudización de conflictos entre los actores, abriendo un espacio de diálogo que habilita nuevas formas de acción política por parte de las instituciones. Además, es preciso subrayar lo siguiente: la perspectiva teórica que sostiene este trabajo se orienta a considerar que el CPRES se instituye con la intención de mejorar ciertas "deficiencias" del funcionamiento del sistema, introduciendo para ello cambios en las prácticas organizacionales. Cuestión ésta altamente compleja en un país como Argentina, caracterizado por la preeminencia de rígidas oposiciones políticas, partidarias, sociales, etcétera, en donde el debate centralización-descentralización ha tomado fuerza en los últimos años con las reformas de las estructuras educativas. Precisamente, la incorporación de una figura como el CPRES hace palmario este problema; su funcionamiento supone un desafío para la definición de políticas educativas, porque plantea un cambio cultural organizacional de agregación de voces, tensiones y consensos, que se procesan en el marco de la interacción interinstitucional.

El CPRES no ha logrado constituirse como una efectiva estructura de implementación. Pero el curso de sus acciones y decisiones influyen en la definición de las políticas educativas, afectando así la performance del sistema.

Finalmente, en un escenario marcado por la escasez de los recursos públicos y la expansión institucional del sistema, tanto el Estado nacional como las instituciones universitarias asumen un rol central en la conducción del sistema, en términos de gobernabilidad. En ese sentido, comienzan a visualizar la necesidad de actuar en forma activa y coordinada, colaborando en la definición de áreas de vacancia y de demandas socioeconómicas para la creación de nueva oferta universitaria.

La capacidad estatal desplegada por el CPRES ha requerido de dos condiciones esenciales para su funcionamiento: la capacidad de conducción técnico-política en el nivel ministerial y de generar reglas, rutinas organizacionales (March y Olsen, 
1997) y roles que dan origen a una identidad organizacional, con agentes que tienen claramente asignado un conjunto de responsabilidades y deberes necesarios para el buen funcionamiento de la organización, y por otra parte, de la capacidad técnico-operativa para promover, integrar y llevar a cabo las propuestas de política ideadas en el interior del complejo universo de la educación superior.

\section{Referencias}

Acuña, Carlos y Fabián Repetto (2006), "La institucionalidad de las políticas y los programas de reducción de la pobreza en América Latina", Informe presentado al Banco Interamericano de Desarrollo (BID).

Aguilar Villanueva, Luis (2007) "El aporte de la política pública y la nueva Gestión pública a la gobernanza", ponencia presentada en el XII Congreso Internacional del CLAD sobre la Reforma del Estado y de la Administración Pública, Santo Domingo, 30 octubre-2 noviembre.

Bobbio, Norberto (1991), Estado, gobierno y sociedad, México, FCE.

CabreroMendoza,Enrique(2006), "Deladescentralización como aspiración, a la descentralización como problema.

El reto de la coordinación intergubernamental en las políticas sociales", Documento presentado en el Seminario sobre Coordinación y Políticas Sociales, Banco Interamericano de Desarrollo, mayo.

Camou, Antonio (2007), "Los juegos de la evaluación universitaria en la Argentina. Notas sobre las interacciones conflictivas entre Estado y universidad", en P. Krostch et al., Evaluando la evaluación. Politicas universitarias, instituciones y actores en Argentina y América Latina, Buenos Aires, Prometeo.

Camou, Antonio (2005), El saber detrás del trono. Intelectuales-expertos, tanques de pensamiento y politicas económicas en la Argentina: entre el "Plan Austral" y la crisis de la Convertibilidad, http://www.antoniocamou.com. ar/El\%20Saber\%20detras\%20del\%20Trono-Camou. htm [consulta: 10 de diciembre, 2009].
Camou,Antonio(2002), "Reformasestatalesde'segunda generación' y reformas universitarias en la Argentina actual ( $\mathrm{O}$ de por qué es bastante más fácil privatizar una línea aérea que reformar una universidad", en Pedro Krotsch, La universidad cautiva: Legados, marcas $y$ horizontes, Buenos Aires, Ediciones Al Margen. Camou, Antonio (2001), "Partidos políticos y gobernabilidad democrática en el México del Postajuste (o de cómo ejercer democráticamente el gobierno y no morir en el primer intento", en J. Labastida y A. Camou, Globalización, identidad y democracia: México y América Latina, México, FLACSO/IIS-UNAM/Siglo XXI.

Catenazzi, A. et al. (2009), El retorno de lo político a la cuestión urbana, Universidad Nacional de General Sarmiento (UNGS), Buenos Aires, Prometeo.

García de Fanelli, Ana María (2009), Profesión académica en la Argentina: carrera e incentivos a los docentes en las universidades nacionales, Buenos Aires, CEDES.

García de Fanelli, Ana María (2005), Universidad, organización e incentivos. Desafios de la politica de financiamiento frente a la complejidad institucional, Buenos Aires, Miño y Dávila.

Gvirtz, Silvina y Gustavo Dufour (2008), Equidad y niveles intermedios de gobierno en los sistemas educativos. Un estudio de casos en la Argentina, Chile, Colombia y Perú, Buenos Aires, Aique.

Krotsch, Pedro y Daniela Atairo (2008), De la proliferación de títulos y el desarrollo disciplinario en las universidades argentinas, www.iipe-buenosaires.org. $\mathrm{ar} /$ publicaciones [consulta: 24 de noviembre, 2009]. 
Krotsch, Pedro et al. (2007), Evaluando la evaluación. Politicas universitarias, instituciones y actores en Argentina y América Latina, Buenos Aires, Prometeo.

Krotsch, Pedro (2001), Educación superior y reformas comparadas, Buenos Aires, Universidad Nacional de Quilmes.

Luhmann, Niklas (1993), Teoría de la sociedad, México, Universidad de Guadalajara Universidad Iberoamericana.

Luhmann, Niklas (1991), Sistemas sociales, México, Alianza.

March, James y Joan Olsen (1997), El redescubrimiento de las organizaciones, México, FCE.

Morlino, Leonardo (1985), Como cambian los regímenes politicos, Madrid, Centro de Estudios Constitucionales.

North, Douglass (2001), Instituciones, cambio institucional $y$ desempeño económico, Buenos Aires, FCE.

Peters, Guy (2006), "Concepts and theories of horizontal policy management", en G. Peters y J. Pierre, Handbook of public administration, Londres, SAGE.

Pérez Rasetti, Carlos (2010), "La expansión de la educación universitaria: políticas y lógicas" (mimeo), documento inédito.

Pérez Rasetti, Carlos (2007), "La expansión geográfica de las universidades y la regulación de las sedes distantes", http://www.me.gov.ar/spu/ [consultado: 23 de marzo, 2009].

Powell, Walter y Paul DiMaggio (1991), The new institutionalism in organizational analysis, Chicago y Londres, The University of Chicago Press.

Pressman, Jeffrey y Aaron Wildavsky (1998), Implementación, México, FCE.
Rapoport, Mario (2000), Historia económica, politica y social de la Argentina, Buenos Aires, Ediciones Macchi.

Repetto, Fabián (2004), "Capacidad estatal. Requisito para el mejoramiento de la política social en América Latina", Departamento de integración y programas regionales, Instituto Interamericano para el Desarrollo social, BID.

Repetto, Fabián (1997), Capacidad estatal y politicas frente a la pobreza: los casos de Chile y Argentina (19901996), tesis doctoral, México, FLACSO.

Romero, Luis Alberto (2003), La crisis Argentina. Una mirada al siglo $X X$, Buenos Aires, Siglo XXI.

Santori, Diego (2005), "El juego político en torno al financiamiento de la universidad pública. El caso de la gestión del gobierno de la Alianza durante el 2001", tesis de maestría en Educación de la Universidad de San Andrés, Buenos Aires.

Secretaría de Políticas Universitarias (SPU), http:// www.me.gov.ar/spu/Servicios/Autoridades_ Universitarias/autoridades_universitarias.html [consulta: 11 de marzo, 2010].

Suasnábar, C. y Rovelli, L. (2009), "Mapeo del Sistema de Educación Superior en el Área Metropolitana de Buenos Aires. Algunas consideraciones sobre su expansión y coordinación", en VI Encuentro Nacional y III Latinoamericano "La Universidad como objeto de investigación", Argentina, Universidad Nacional de Córdoba.

Winter, Soren (2006) "Implementation" en G. Peters y J. Pierre, Handbook of public administration, Londres, SAGE. 


\section{Fuentes normativas consultadas:}

Decreto 1047/99, www.me.gov.ar/spu/guia_tematica/CPRES/cpres-normativas.htm [consulta: 10 de junio, 2009].

Decreto 576/96, www.me.gov.ar/spu/guia_tematica/CPRES/cpres-normativas.htm [consulta: 10 de junio, 2009].

Decreto 451/73, http://www.infoleg.gov.ar/infolegInternet/verNorma.do?id=23743

[consulta: 10 de junio, 2009].

Decreto 499/95, http://www.uncu.edu.ar/upload/decreto_nro_499-05.pdf

[consulta: 10 de junio, 2009].

Decreto 282/93, www.me.gov.ar/spu/guia_tematica/CPRES/cpres-normativas.htm [consulta: 10 de junio, 2009].

Ley de Educación Superior 24.521, Sancionada el 20 de Julio de 1995, Publicada el 10 de Agosto de 1995

(Boletín Oficial 28.204), Argentina.

Resolución 602/95, www.me.gov.ar/spu/guia_tematica/CPRES/cpres-normativas.htm [consulta: 27 de junio, 2009].

Resolución 200/96, www.me.gov.ar/spu/guia_tematica/CPRES/cpres-normativas.htm [consulta: 27 de junio, 2009].

\section{Cómo citar este artículo:}

González, Giselle (2011), "La territorialización de las políticas públicas en Argentina. Un estudio acerca del CPRES en el área metropolitana, en Revista Iberoamericana de Educación Superior (RIES), México, ISSUE-UNAM/Universia, vol. II, núm. 4, pp. 41-61, http://ries.universia.net/index.php/ries/article/view/49 [consulta: fecha de última consulta]. 